\title{
Charm production in antiproton-proton annihilation ${ }^{\star}$
}

\author{
J. Haidenbauer • G. Krein
}

Received: date / Accepted: date

\begin{abstract}
We study the production of charmed mesons $(D)$ and baryons $\left(\Lambda_{c}\right)$ in antiproton-proton $(\bar{p} p)$ annihilation close to their respective production thresholds. The elementary charm production process is described by either baryon/meson exchange or by quark/gluon dynamics. Effects of the interactions in the initial and final states are taken into account rigorously. The calculations are performed in close analogy to our earlier study on $\bar{p} p \rightarrow \bar{\Lambda} \Lambda$ and $\bar{p} p \rightarrow \bar{K} K$ by connecting the processes via SU(4) flavor symmetry. Our predictions for the $\bar{\Lambda}_{c} \Lambda_{c}$ production cross section are in the order of 1 to $7 \mathrm{mb}$, i.e. a factor of around 10-70 smaller than the corresponding cross sections for $\bar{\Lambda} \Lambda$ However, they are 100 to 1000 times larger than predictions of other model calculations in the literature. On the other hand, the resulting cross sections for $\bar{D} D$ production are found to be in the order of $10^{-2}-10^{-1} \mu b$ and they turned out to be comparable to those obtained in other studies.
\end{abstract}

PACS 13.75.Cs $\cdot$ 13.85.Fb $\cdot 25.43 .+\mathrm{t}$

\section{Introduction}

The study of the production of charmed hadrons in antiproton-proton $(\bar{p} p)$ collisions is of importance for the understanding of the strong force in the nonperturbative regime of QCD. The FAIR project at the GSI site has an extensive program aiming at a highaccuracy spectroscopy of charmed hadrons and at an investigation of their interactions with ordinary matter 1]. Presently little is known about such interactions and their knowledge is a prerequisite for investigating issues like in-medium properties of charmed

* Presented at the 21st European Conference on Few-Body Problems in Physics, Salamanca, Spain, 30 August - 3 September 2010.

J. Haidenbauer

Institute for Advanced Simulation, Forschungszentrum Jülich, D-52425 Jülich, Germany

E-mail: j.haidenbauer@fz-juelich.de

G. Krein

Instituto de Física Teórica, Universidade Estadual Paulista,

Rua Dr. Bento Teobaldo Ferraz, 271 - 01140-070 São Paulo, SP, Brazil

E-mail: gkrein@ift.unesp.br 
hadrons, e.g. $c \bar{c}$-quarkonium dissociation and changes in properties of $D$ mesons due to chiral symmetry restoration effects on the light quarks composing these mesons.

In this contribution we present predictions for the charm-production reactions $\bar{p} p \rightarrow$ $\bar{\Lambda}_{c}^{-} \Lambda_{c}^{+}$and $\bar{p} p \rightarrow \bar{D} D$ close to their respective thresholds 2,3 . The work builds on the Jülich meson-baryon models for the reactions $\bar{p} p \rightarrow \bar{\Lambda} \Lambda$ [4] and $\bar{p} p \rightarrow \bar{K} K$ [5]. The extension of those models from the strangeness to the charm sector follows a strategy similar to our recent work on the $D N$ and $\bar{D} N$ interactions 6, 6] namely by assuming as a working hypothesis $\mathrm{SU}(4)$ symmetry constraints. We also compare our results with those of other model calculations in the literature 8, 10,11, 12. In some of these studies a quark-gluon description based on a factorization hypothesis of hard and soft processes 8,12 is employed, while in others a non-perturbative quark-gluon string model is used, based on secondary Regge pole exchanges including absorptive corrections 9,11 .

\section{The model}

The calculations of the charm-production reactions $\bar{p} p \rightarrow \bar{\Lambda}_{c}^{-} \Lambda_{c}^{+}$and $\bar{p} p \rightarrow \bar{D} D$ are done in complete analogy to past investigations of the strangeness-production reactions $\bar{p} p \rightarrow \bar{\Lambda} \Lambda$ [4] and $\bar{p} p \rightarrow \bar{K} K\left[5\right.$ ] by the Jülich group. In particular $\bar{p} p \rightarrow \bar{\Lambda}_{c}^{-} \Lambda_{c}^{+}$is performed within a coupled-channel approach while for $\bar{p} p \rightarrow \bar{D} D$ a DWBA approach is employed. This allows us to take into account rigorously the effects of the initial $(\bar{p} p)$ and also of the final state interactions which play an important role for energies near the production threshold [4, 13.

Because of the known sensitivity of the results for the cross sections on the initial $\bar{p} p$ interaction we examine its effect by considering several variants of the $\bar{N} N$ potential. Details of those potentials can be found in Ref. 2, 3. Here we just want to mention that they differ primarily in the elastic part where we consider variations from keeping only the longest ranged contribution (one-pion exchange) to taking a full G-parity transformed $N N$ interaction as done in [4. All these models reproduce the total $\bar{p} p$ cross section in the relevant energy range and, in general, describe also data on integrated elastic and charge-exchange cross sections and even $\bar{p} p$ differential cross sections, cf. [2].

The microscopic charm-production processes are described by either meson exchange $\left(D, D^{*}\right)$ in case of $\bar{p} p \rightarrow \bar{\Lambda}_{c}^{-} \Lambda_{c}^{+}$, or baryon exchange $\left(\Lambda_{c}, \Sigma_{c}\right)$ for $\bar{p} p \rightarrow \bar{D} D$. The transition potentials are derived from the corresponding transitions in the strangenessproduction channels $(\bar{\Lambda} \Lambda, \bar{K} K)$ under the assumption of SU(4) symmetry. In order to assess uncertainties in the model we study, in addition, the effect of replacing the meson-exchange transition potential by a charm-production mechanism derived in a quark model [2,13].

\section{Results for the reaction $\bar{p} p \rightarrow \bar{\Lambda}_{c}^{-} \Lambda_{c}^{+}$}

Predictions for the total reaction cross section for $p \bar{p} \rightarrow \bar{\Lambda}_{c}^{-} \Lambda_{c}^{+}$are presented in Fig. 1 In the left panel the cross section is shown as a function of the excess energy $\epsilon=\sqrt{s}-$ $m_{\Lambda_{c}}-m_{\bar{\Lambda}_{c}}$ so that we can compare it with the one for $p \bar{p} \rightarrow \bar{\Lambda} \Lambda$ at the corresponding $\epsilon$. The curve in Fig. 1 correspond to the $\bar{p} p$ model C 2 .

Obviously, and as expected, the cross section for $\bar{\Lambda}_{c}^{-} \Lambda_{c}^{+}$production is smaller than the one for $\bar{\Lambda} \Lambda$. But the difference is about one order of magnitude only. We display 

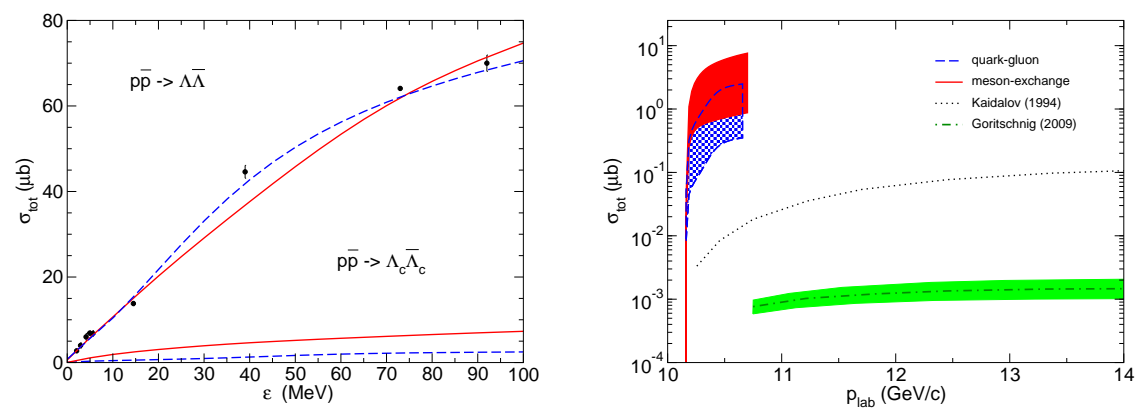

Fig. 1 Left: Total reaction cross sections for $\bar{p} p \rightarrow \bar{\Lambda} \Lambda$ and $\bar{p} p \rightarrow \bar{\Lambda}_{c}^{-} \Lambda_{c}^{+}$as a function of the excess energy $\epsilon$. The solid curves are results for the meson-exchange transition potential while the dashed curves correspond to quark-gluon dynamics. Right: Total reaction cross sections for $\bar{p} p \rightarrow \bar{\Lambda}_{c}^{-} \Lambda_{c}^{+}$as a function of $p_{l a b}$. Our predictions (shaded band, grid) are compared to those by Refs. [9] and [12].

here also the results based on an adaption of the ${ }^{3} S_{1}$ quark-gluon transition mechanism of Ref. 13 . We scale the effective quark-gluon coupling strength, fixed in our study of $\bar{p} p \rightarrow \bar{\Lambda} \Lambda$ [4, with $\left(m_{c} / m_{s}\right)^{2}$ using the constituent quark masses $m_{s}=550 \mathrm{MeV}$ and $m_{c}=1600 \mathrm{MeV}$, i.e. the same values as employed in our previous works in Ref. 6]. As expected, we obtain cross sections that are of the same magnitude as those predicted in the meson-exchange picture though roughly a factor three smaller, cf. the dashed line in Fig. 1.

On the right-hand side of Fig. 1 we compare our predictions with those by other groups 9, 12]. Our results are shown as bands in order to reflect the variation of the predictions when different ISI's are used. The dark (red) shaded band and the (blue) grid correspond to results based on the meson-exchange and quark-gluon transition potentials, respectively. It is remarkable that our results differ drastically from those of the preceeding works. Specifically, our cross sections are a factor 1000 larger than those given in [12] and they are still about 100 times larger than the ones in 9]. Thus, even when considering the variation of about a factor ten due to the ISI that we see in our results and the uncertainties due to the unconstrained FSI and form factors in the transition potential that amount to roughly a factor three 2, we are faced with an impressive qualitative difference.

\section{Results for the reaction $\bar{p} p \rightarrow \bar{D} D$}

Our predictions for the reaction $\bar{p} p \rightarrow \bar{D} D$ are presented in Fig. 2. Let us first focus on the effects of the inital- and final state interaction. The transition from $\bar{p} p$ to $\bar{D} D$ is generated by the exchange of charmed baryons, in particular the $\Lambda_{c}$ and $\Sigma_{c}$. Under the assumption of $S U(4)$ symmetry the pertinent coupling constants are given by

$$
\begin{aligned}
& f_{\Lambda_{c} N D}=-\frac{1}{\sqrt{3}}(1+2 \alpha) f_{N N \pi} \approx-1.04 f_{N N \pi}, \\
& f_{\Sigma_{c} N D}=(1-2 \alpha) f_{N N \pi} \approx 0.2 f_{N N \pi},
\end{aligned}
$$



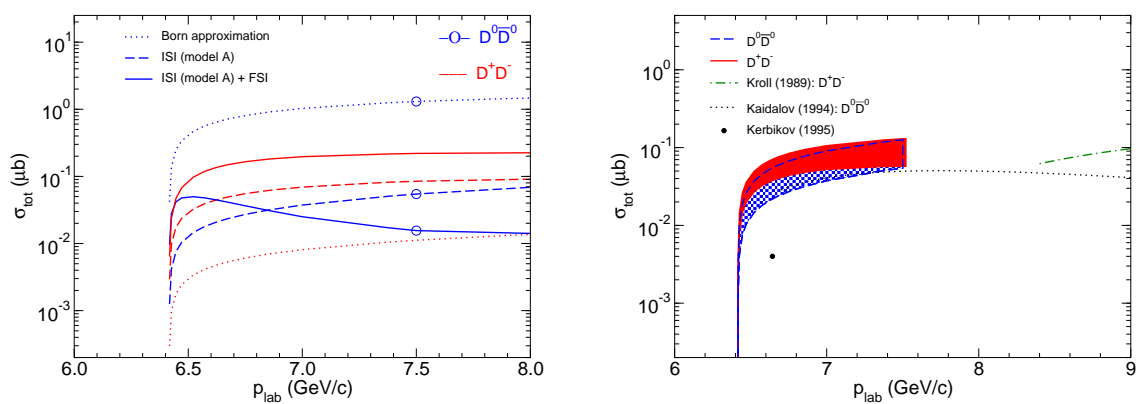

Fig. 2 Total reaction cross sections for $\bar{p} p \rightarrow \bar{D} D$ as a function of $p_{l a b}$. Left: Effects of the initial and final state interaction. Right: Our predictions (shaded band, grid) in comparison to those of Refs. 8], 9] and [10.

where we assumed for the $\mathrm{F} /(\mathrm{F}+\mathrm{D})$ ratio $\alpha \approx 0.4$. Thus, one expects that $\Lambda_{c}$ exchange dominates the transition while $\Sigma_{c}$ exchange should be suppressed. Specifically, this implies that $V^{\bar{p} p \rightarrow D^{0} \bar{D}^{0}} \gg V^{\bar{p} p \rightarrow D^{+} D^{-}}$. Indeed, within the Born approximation the cross sections predicted for $D^{0} \bar{D}^{0}$ are more than two orders of magnitude larger than those for $D^{+} D^{-}$11], cf. the dotted lines Fig. 2 (The curves for $D^{0} \bar{D}^{0}$ are marked with a circle.) However, once the ISI is included the situation changes drastically and now both channels are produced at a comparable rate (dashed lines). In fact, now the cross section for $D^{+} D^{-}$is even somewhat larger than the one for $D^{0} \bar{D}^{0}$. The inclusion of the FSI modifies the predictions once again, though only on a quantitative level.

On the right-hand side of Fig. 2 we compare our predictions with those by other groups. Here our results are shown as a shaded band for $D^{+} D^{-}$and as grid for $D^{0} \bar{D}^{0}$. Again the bands represent the variation of the predictions when different ISI's are used. Displayed are also the predictions by Kroll et al. [8] (dash-dotted line), Kaidalov [9] (dotted line) and by Kerbikov [10] (circle). Obviously, and unlike in the $\bar{\Lambda}_{c}^{-} \Lambda_{c}^{+}$ case, now the majority of the predictions are pretty much comparable, at least on a qualitative level.

\section{Summary}

We have presented results for the reaction $\bar{p} p \rightarrow \bar{\Lambda}_{c}^{-} \Lambda_{c}^{+}$of a model calculation performed within the meson-exchange picture in close analogy to the Jülich analysis of the reaction $\bar{p} p \rightarrow \bar{\Lambda} \Lambda$ utilizing $S U(4)$ symmetry. The predicted cross sections are in the order of $1-7 \mu b$. Thus, they are about 10-100 times smaller than those for $\bar{p} p \rightarrow \bar{\Lambda} \Lambda$. However, and surprisingly, our predictions turned out to be about 1000 times larger than those obtained in other model calculations.

We presented also predictions for $\bar{p} p \rightarrow \bar{D} D$ obtained in the same spirit, i.e. by connecting this reaction with $\bar{p} p \rightarrow \bar{K} K$ via $S U(4)$ symmetry. Here the cross sections were found to be in the order of $10^{-2}-10^{-1} \mu b$ and they turned out to be comparable to those predicted by other model calculations.

Acknowledgements This work was partially financed by CNPq and FAPESP (Brazilian agencies). 


\section{References}

1. W. Erni et al. (Panda Collaboration), arXiv:0903.3905 [hep-ex].

2. J. Haidenbauer and G. Krein, Phys. Lett. B 687, 314 (2010).

3. J. Haidenbauer and G. Krein, in preparation.

4. J. Haidenbauer et al., Phys. Rev. C 45, 931 (1992); Phys. Rev. C 46 (1992) 2158.

5. V. Mull and K. Holinde, Phys. Rev. C 51, 2360 (1995)

6. J. Haidenbauer, G. Krein, U.-G. Meißner and A. Sibirtsev, Eur. Phys. J. A 33, 107 (2007);

Eur. Phys. J. A 37, 55 (2008).

7. J. Haidenbauer, G. Krein, U.-G. Meißner and L. Tolos, arXiv:1008.3794 [nucl-th]

8. P. Kroll, B. Quadder and W. Schweiger, Nucl. Phys. B 316, 373 (1989).

9. A. B. Kaidalov and P. E. Volkovitsky, Z. Phys. C 63, 517 (1994).

10. B. Kerbikov and D. Kharzeev, Phys. Rev. D 51, 6103 (1995).

11. A. I. Titov and B. Kämpfer, Phys. Rev. C 78, 025201 (2008).

12. A. T. Goritschnig, P. Kroll and W. Schweiger, Eur. Phys. J. A 42, 43 (2009).

13. M. Kohno and W. Weise, Phys. Lett. B 179, 15 (1986). 


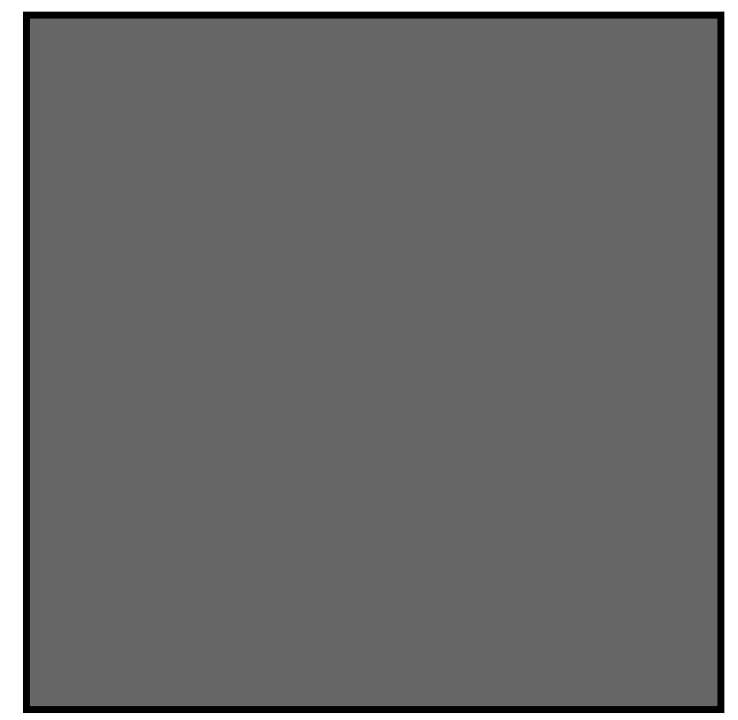

